\author{
Monique Brink \\ Jaap Deunk \\ Helena M. Dekker \\ Michael J. R. Edwards \\ Digna R. Kool \\ Arie B. van Vugt \\ Cornelis van Kuijk \\ Johan G. Blickman
}

\section{Criteria for the selective use of chest computed tomography in blunt trauma patients}

Received: 20 May 2009

Revised: 7 August 2009

Accepted: 13 August 2009

Published online: 17 September 2009

(C) The Author(s) 2009.

This article is published with open access at Springerlink.com

Trial number: NCT00228111

at www.clinicaltrials.gov

M. Brink $(\bowtie) \cdot$ H. M. Dekker ·

D. R. Kool - J. G. Blickman

Department of Radiology,

Radboud University Nijmegen

Medical Centre,

Geert Grooteplein 10, Internal no.

(Huispost) 667,

PO Box: 9101, $6500 \mathrm{HB}$

Nijmegen, the Netherlands e-mail: M.brink@rad.umcn.nl Tel.: +31-24-3540866

J. Deunk · M. J. R. Edwards Department of Surgery,

Radboud University Nijmegen

Medical Centre,

Geert Grooteplein 10, Internal no.

(Huispost) 690,

PO Box: 9101, $6500 \mathrm{HB}$

Nijmegen, the Netherlands

\author{
A. B. van Vugt \\ Department of Emergency Medicine, \\ Radboud University Nijmegen Medical \\ Centre Nijmegen, \\ Geert Grooteplein 10, Internal no. \\ (Huispost) 690, \\ PO Box: 9101, $6500 \mathrm{HB}$ \\ Nijmegen, the Netherlands \\ C. van Kuijk \\ Department of Radiology, \\ VU (Vrije Universiteit) University \\ Medical Center Amsterdam, \\ Internal no. PKOX134, \\ PO Box: 7057, 1007 MB \\ Amsterdam, the Netherlands
}

Abstract Purpose: The purpose of
this study was to derive parameters
that predict which high-energy blunt
trauma patients should undergo com-
puted tomography (CT) for detection
of chest injury. Methods: This
observational study prospectively
included consecutive patients
( $\geq 16$ years old) who underwent
multidetector CT of the chest after a
high-energy mechanism of blunt
trauma in one trauma centre.
Results: We included 1,047 patients
(median age, $37 ; 70 \%$ male), of whom
508 had chest injuries identified by CT. Using logistic regression, we identified nine predictors of chest injury presence on CT (age $\geq 55$ years, abnormal chest physical examination, altered sensorium, abnormal thoracic spine physical examination, abnormal chest conventional radiography (CR), abnormal thoracic spine CR, abnormal pelvic CR or abdominal ultrasound, base excess $<-3 \mathrm{mmol} / 1$ and haemoglobin $<6 \mathrm{mmol} / \mathrm{l}$ ). Of 855 patients with $\geq 1$ positive predictors, 484 had injury on CT ( $95 \%$ of all 508 patients with injury). Of all 192 patients with no positive predictor, $24(13 \%)$ had chest injury, of whom $4(2 \%)$ had injuries that were considered clinically relevant. Conclusion: Omission of CT in patients without any positive predictor could reduce imaging frequency by $18 \%$, while most clinically relevant chest injuries remain adequately detected.

Keywords Blunt trauma . Computed tomography · Thorax . Wounds and injuries .

Logistic models $\cdot$ Prediction rules

\section{Introduction}

Technological advances in computed tomography (CT) have made chest CT a fast and accurate, and therefore extensively used imaging technique in trauma patient care $[1,2]$.

Although the utility of CT for detection of chest injuries is primarily demonstrated in severely injured patients, CT is now increasingly being used in the less severely injured trauma population [3]. This widespread use deserves reconsideration because its effectiveness might not always outweigh potential harm by radiation exposure $[4,5]$, medicalisation, time loss [6] and the high costs.

Although many studies addressed the value of CT in trauma patients, few evidence-based indications for trauma CT of the chest exist [7]. According to the American College of Radiology appropriateness criteria, CT should be performed if conventional radiography (CR) shows 
signs of mediastinal bleeding suspicious for blunt aortic injury [8]. These guidelines additionally state that thoracic spine images are now effectively obtained in all patients who undergo thoracoabdominal CT, making indications for spine imaging less important than indications for obtaining thoracoabdominal CT [9]. The Eastern Association for the Surgery of Trauma guidelines summarise that the thoracolumbar spine can be cleared without imaging in awake trauma patients without any evidence of intoxication with ethanol or drugs, who have a normal mental status and normal physical examinations [10]. However, these guidelines also state that aortic injuries cannot be accurately ruled out by using signs of mediastinal bleeding on CR. They therefore suggest that blunt aortic injury should be considered in all patients involved in motor vehicle collisions [11]. These recommendations reflect that little evidence exists on which patients are likely to benefit from chest CT after blunt trauma. More importantly, it remains unclear in which patients chest CT can be omitted without missing relevant injuries.
The aim of this prospective study on adult blunt trauma patients is therefore to derive a set of independent clinical parameters that distinguish patients who will benefit from chest $\mathrm{CT}$ from patients in whom chest $\mathrm{CT}$ can be omitted without missing relevant injuries.

\section{Patients and methods}

\section{Patients}

We performed an observational cohort study on 1,047 consecutive blunt trauma patients who were 16 years and older [12]. Patients were prospectively included according to the inclusion and exclusion criteria in Table 1 if they were primarily evaluated at the emergency department of our hospital from May 2005 until July 2008.

All patients underwent the same diagnostic protocol according to our hospital's guidelines. This protocol consisted of physical examination (PE), CR of the chest,

Table 1 Inclusion and exclusion criteria defined before the study started [12]

\begin{tabular}{|c|c|}
\hline Inclusion criteria $^{a}$ & Definitions \\
\hline \multicolumn{2}{|l|}{ Life-threatening vital problems due to trauma } \\
\hline -Airway patency problems & As declared by anaesthesiologist \\
\hline -Breathing problems & Breathing frequency $>30 / \mathrm{min}$ \\
\hline -Circulatory problems & Pulse $>120 / \mathrm{min}$, systolic blood pressure $<100 \mathrm{mmHg}$, capillary refill $>4 \mathrm{~s}$ \\
\hline -Neurological problems & $\begin{array}{l}\text { Exterior blood loss }>500 \mathrm{ml} \\
\mathrm{GCS}^{\mathrm{b}} \leq 13\end{array}$ \\
\hline \multicolumn{2}{|l|}{ Clinical evidence of serious injuries } \\
\hline -Clinically evident pelvic ring fracture & As declared by attending surgeon \\
\hline $\begin{array}{l}\text {-Clinical signs of unstable vertebral fractures or spinal cord } \\
\text { compression }\end{array}$ & As declared by attending surgeon \\
\hline \multicolumn{2}{|l|}{ Severe mechanism of injury } \\
\hline $\begin{array}{l}\text {-High-energy mechanism of injury as declared by pre-hospital } \\
\text { emergency medical services }\end{array}$ & $\begin{array}{l}\text { Fall from height }>3 \mathrm{~m} \\
\text { Motor vehicle accident }>50 \mathrm{~km} / \mathrm{h} \\
\text { Ejection from vehicle } \\
\text { Car rollover } \\
\text { Severe impact damage to car } \\
\text { Struck pedestrian }>10 \mathrm{~km} / \mathrm{h} \\
\text { Struck bicyclist }>30 \mathrm{~km} / \mathrm{h}\end{array}$ \\
\hline -High-energy crush injury to torso & Squeezed under or between heavy objects \\
\hline \multicolumn{2}{|l|}{ Exclusion criteria } \\
\hline \multicolumn{2}{|l|}{ CT not feasible/appropriate } \\
\hline -Dead soon after arrival & Within \pm 15 min of arrival, as declared by attending surgeon \\
\hline -Shock class III/IV & $\begin{array}{l}\text { Pulse rate }>120 / \text { min or systolic blood pressure }<100 \mathrm{mmHg} \text { and non } \\
\text { respondent to volume therapy }\end{array}$ \\
\hline -Immediate (neuro) surgical intervention & As declared by neurosurgeon \\
\hline -Pregnancy & Suspected by history or sonography \\
\hline
\end{tabular}

Notes: ${ }^{a}$ For inclusion in the study, only one criterion had to be met. ${ }^{b} \mathrm{GCS}$, Glasgow Coma Scale 
spine and pelvis, abdominal ultrasonography and CT of the cervical spine, chest, abdomen, spine and pelvis.

$\mathrm{PE}$ was performed and documented by residents in surgery or orthopaedics or emergency physicians who were supervised by senior trauma surgeons according to the Advanced Trauma Life Support ${ }^{\circledR}$ guidelines [13]. Blood samples were collected for laboratory investigations including arterial blood gas analysis, haematological measurements and biochemistry.

CR was executed on a vertix 3D system (Siemens Medical Solutions, Forchheim, Germany) and consisted of a supine view of the chest and pelvis in an anteroposterior direction and of the thoracolumbar spine in an anteroposterior and lateral projection. Abdominal ultrasonography was primarily used to detect or exclude free intraperitoneal fluid according to the principles of focused ultrasonography for trauma. CR and ultrasonography were interpreted immediately by senior residents in radiology and the supervising trauma radiologists.

All patients thereafter underwent CT on a 16-detector unit (Somatom Sensation 16, Siemens Medical Solutions, Forchheim, Germany) that was located in the emergency department. CT of the cervical spine was obtained from the occipital condyles to the first thoracic vertebra. Chest CT was performed as a part of a thoracoabdominal examination from the acromioclavicular joint to the lesser trochanter, with automated exposure control at a reference effective tube current time product of $200 \mathrm{mAs}$, a tube voltage of $120 \mathrm{kV}$, a beam collimation of $16 \times 1.5 \mathrm{~mm}$, a median dose-length product of $1,150 \mathrm{mGycm}$ and administration of $100 \mathrm{ml}$ of intravenous contrast agent [Iobitridol $300 \mathrm{mg} \mathrm{I} / \mathrm{ml}$ (Xenetix 300, Guerbet, Paris, France)]. Reconstructed section thickness was $3 \mathrm{~mm}$ in a bone, lung and soft tissue setting and section overlap was 1.5, 3 and $3 \mathrm{~mm}$, respectively. Finally, sagittal and coronal multiplanar reformatted images of the spine were constructed. After review by radiology residents supervised by certified radiologists, the trauma team started or changed patient management as needed. Finally, an effort was made to follow every patient for 6 months, either by medical consultation in the outpatient clinics or by telephone.

The institutional ethics board approved the study protocol. The requirement for informed consent could be waived because this was an observational study of a standard diagnostic protocol, and all patients received the same type of diagnostics and care.

\section{Data collection}

Two unblinded investigators (with 1 and 3 years' experience in emergency medicine at the start of the study) attended briefings and resuscitations of included patients and reviewed all charts and radiological reports. They collected data on patient characteristics, diagnoses and treatment by using standardised abstraction forms. These data had all been prospectively recorded by the trauma team members before CT was performed. If necessary, the investigators made a subsequent request for additional information from trauma team members. The investigators re-reviewed all patients' charts after 6 months to establish whether injuries were initially missed. They collected injury severity scores (ISS) from the regional trauma registry and finally imported all data into a customised database.

\section{Outcome measures}

Two outcome measures were determined before this study started: (1) presence of chest injuries on CT and (2) clinically relevant occult injuries.

Chest injuries on chest CT consisted of aortic injury, diaphragmatic injury, tracheobronchial tree injury, oesophageal injury, pneumothorax, haemothorax and pulmonary contusion. They also consisted of fractures of the ribs, scapula, sternum and thoracic vertebrae (including the vertebral body and the transverse and spinous processes). The presence of these injuries was recorded per patient. If pneumothoraces, pulmonary contusions, haemothoraces or rib fractures were present, the investigators recorded their extent (number), location and severity (minimal, moderate and severe). These classifications were based on a consensus reading of 54 cases that were not included in this study.

Clinically relevant occult injuries were defined as injuries on $\mathrm{CT}$ that were not visualised by $\mathrm{CR}$ of the chest and thoracic spine and that had an impact on patient management. An impact on patient management was defined as the occurrence of changes in treatment as a direct result of the CT findings. These changes were determined before the study started and included additional diagnostic workup, changes in intensity of care (care level upgrade) and immediate interventions that were started by the trauma team.

\section{Predictor variables}

We selected dichotomous candidate predictors of injuries on CT based on a review of the literature and clinical experience. These variables could be determined during initial patient evaluation at the emergency department and were derived from pre-hospital service reports, emergency records, radiological reports of CR and abdominal ultrasonography investigations and blood sample analyses.

In the literature, cervical spine fractures have been associated with thoracic spine injuries [14]. However, because cervical spine $\mathrm{CT}$ reconstructions were not readily available before chest CT was obtained, we did not consider presence of cervical spine injuries a practical predictor of chest injuries in this setting. 


\section{Statistical analysis}

In this study, we primarily aimed to distinguish patients with injuries on chest $\mathrm{CT}$ from patients without injuries on chest $\mathrm{CT}$ by taking the following steps.

First, candidate predictors of injuries on CT were combined into dichotomous composite predictors based on clinical similarity and strong biological association (Table 2). If data were missing or incomplete, these were imputed as "normal".

Second, univariate logistic regression analysis was used to study the ability of each composite predictor to distinguish patients with injuries from patients without injuries on chest CT. Crude odds ratios (OR) of all positive composite predictors were derived for the dependent variable "presence of injuries on CT (yes, no)".

Third, multivariate regression with a forward selection procedure [15] was used to identify independent composite predictors of presence of injuries on chest CT. A priori, we forced the composite predictor "altered sensorium" (GCS $<14$, clinical suspicion of drug or alcohol intoxication, orotracheal intubation before clinical evaluation) into the multivariate regression model, because we considered this variable to have great clinical relevance [10]. All other composite predictors that were statistically significantly related to the risk of injuries on $\mathrm{CT}$ in the univariate analysis $(p<0.05$ in the likelihood ratio test) were used as independent variables in the selection procedure. This yielded a regression model in which only statistically significant independent predictors were finally included. This model was checked for collinearity and interaction between variables by incorporating biologically plausible interaction terms in the model. Adjusted odds ratios were presented to indicate the predictive strength of these predictors. Discriminatory power of the final regression model was assessed with the area under the receiver-operating characteristic (ROC) curve (AUC) [15] and the percentage of explained variance with the $\mathrm{R}$-square. To evaluate the reliability of the regression model, an internal validation was performed with a bootstrap analysis and corrected R-square and AUC measures were presented [16].

Our final aim was to construct a predictive model that defines patients in whom CT can be omitted while missing relevant injuries in as few patients as possible. We therefore chose a predicted probability cutoff point on the ROC curve at which the sensitivity for injuries on $\mathrm{CT}$ was as high as possible with a specificity $>0$. Using this cutoff point effectively meant that patients without any positive independent predictor were classified as low-risk patients, whereas patients with any positive independent predictor were all classified as high-risk patients. We evaluated the predictive model with this cutoff point by presenting the model's sensitivity and specificity for presence of chest injuries and for presence of clinically relevant occult chest injuries on $\mathrm{CT}$.
We performed statistical analyses with the statistical packages for Microsoft Windows SPPS, version 16.0 (Chicago, IL), and R, version 2.6.1 (The R Project for Statistical Computing, www.r-project.org).

\section{Results}

From May 2005 until July 2008, 1,199 patients fulfilled the study inclusion criteria. Eighty-one patients were excluded because of predetermined exclusion criteria and 71 patients because of protocol violation; CT was not performed in these patients (Fig. 1).

A total of 1,047 patients were included in this study of whom 731 patients $(70 \%)$ were male. Median age was 37 years (range, 16-95). Median ISS was 14, and mean ISS was 17 (range, 0-75). Five-hundred eight patients (49\%) had injuries visible on CT. In 459 (44\%) patients, CT detected occult injuries (additional injuries compared with CR of the chest). In 183 (17\%) patients, these occult injuries had an impact on patient management and were therefore considered to be clinically relevant. These management changes comprised care level upgrading $(n=60)$, chest drain (re)positioning $(n=45)$, conservative $(n=34)$ or surgical stabilisation $(n=19)$ of spinal fractures, epidural anaesthesia in cases of multiple occult rib fractures $(n=15)$, consultation with cardiologists $(n=14)$, angiography $(n=8)$, bronchoscopy $(n=5)$, interventional radiology (aortic repair, $n=4$, embolisation, $n=1)$, thoracotomy $(n=2)$ and treatment for tracheal $(\mathrm{n}=1)$ or oesophageal rupture $(n=1)$.

Of all included patients, 43 (4\%) were lost to follow-up. Completed follow-up revealed that in one patient with multiple chest injuries, a diaphragmatic rupture was initially missed on CT. This injury was revealed after cessation of ventilation 2 days post-trauma and was treated with a delayed laparotomy with good patient recovery. Conversely, another patient with multiple chest injuries was suspected to have a diaphragmatic injury on CT. However, an emergency laparotomy, which was indicated for a splenectomy, did not confirm this injury. A third patient with multiple serious injuries on chest $\mathrm{CT}$ developed a pericardial tamponade 3 weeks post-trauma. Although CT was therefore not $100 \%$ accurate in the detection of all specific chest injuries, completed clinical follow-up revealed that $\mathrm{CT}$ correctly classified patients as having or not having some injury of the chest.

\section{Predictive model}

Data were complete for all predictors except for blood analyses and CR of the thoracic spine. In 23 patients no haemoglobin and in 258 patients no BE samples were obtained, mainly because patients had no respiratory or haemodynamic problems. In 46 patients, CR of the thoracic 
Table 2 Definitions of composite predictors of chest injuries on CT

\begin{tabular}{|c|c|c|}
\hline Composite predictor & $\begin{array}{l}\text { Definition: predictors were positive if any of the following conditions } \\
\text { were fulfilled }\end{array}$ & References \\
\hline$\geq 55$ years & -Age 55 years or older & {$[28]$} \\
\hline $\begin{array}{l}\text { Dangerous mechanism } \\
\text { of injury }\end{array}$ & $\begin{array}{l}\text { Motor vehicle collision and any of the following: } \\
\text {-No use of constraints } \\
\text {-Ejection from the vehicle } \\
\text {-Death occupant }\end{array}$ & {$[20,21,29-32]$} \\
\hline$P E^{b}$ chest & $\begin{array}{l}\text { - Breathing frequency }<10 / \text { min or }>29 / \text { min (pre-hospital or on } \\
\text { presentation } \\
\text { at the } \mathrm{ED}^{\mathrm{a}} \text { ) } \\
\text {-Pulse oximetry } \mathrm{SaO}_{2}<95 \% \text { at presentation at the ED } \\
\text {-Decreased breathing sounds at auscultation } \\
\text {-Subcutaneous emphysema at palpation } \\
\text {-Tenderness to palpation of the chest wall } \\
\text { - Lacerations or haematoma of the chest wall }\end{array}$ & {$[20-23,33-40]$} \\
\hline PE circulatory problems & $\begin{array}{l}\text {-Systolic blood pressure }<90 \mathrm{mmHg} \text { (pre-hospital or at presentation } \\
\text { at the ED) } \\
\text {-Heart rate }>120 \text { beats per minute (pre-hospital or at presentation } \\
\text { at the ED) }\end{array}$ & {$[20-22,28,41]$} \\
\hline $\mathrm{PE}$ altered sensorium & $\begin{array}{l}\text {-Glasgow coma scale }<14 \text { on initial presentation at the ED } \\
\text {-Orotracheal intubation before clinical evaluation at the ED } \\
\text {-Clinical suspicion of drugs or alcohol intoxication }\end{array}$ & {$[14,22,24,40,42]$} \\
\hline PE supraclavicular injury & $\begin{array}{l}\text {-Any fracture, laceration or haematoma above the clavicle, } \\
\text { including the face }\end{array}$ & {$[20,21]$} \\
\hline PE thoracic spine & $\begin{array}{l}\text {-Tenderness to palpation of the midline of the thoracic spine } \\
\text {-Thoracolumbar lacerations or haematoma } \\
\text {-Neurological deficit suggesting spinal cord injury }\end{array}$ & {$[14,32,42]$} \\
\hline PE abdomen & $\begin{array}{l}\text {-Tenderness to palpation } \\
\text {-Lacerations or haematoma } \\
\text {-Abdominal distension or guarding }\end{array}$ & \\
\hline PE extremity fracture & $\begin{array}{l}\text {-Clinical suspicion of fractures of the upper or lower extremities, } \\
\text { if CR of the extremities were obtained }\end{array}$ & {$[20-22,43,44]$} \\
\hline $\mathrm{CR}^{\mathrm{c}}$ chest & $\begin{array}{l}\text { Any of the following abnormalities identified on CR of the chest } \\
\text {-Pulmonary contusion } \\
\text {-Haemothorax } \\
\text {-Pneumothorax } \\
\text {-Subcutaneous emphysema } \\
\text {-Abnormal mediastinum suggesting aortic injury } \\
\text {-Spinal fracture } \\
\text {-Diaphragmatic rupture } \\
\text {-Rib fractures } \\
\text {-Scapular fracture } \\
\text {-Clavicular fracture }\end{array}$ & {$[2,20,21,38,41,43,45,46]$} \\
\hline CR thoracic spine & $\begin{array}{l}\text { Any of the following abnormalities on } \mathrm{CR} \text { of the thoracic spine: } \\
\text {-Any fracture of the vertebral body or spinous or transverse processes } \\
\text {-Spinal malalignment }\end{array}$ & {$[47]$} \\
\hline
\end{tabular}


Table 2 (continued)

\begin{tabular}{|c|c|c|}
\hline Composite predictor & $\begin{array}{l}\text { Definition: predictors were positive if any of the following conditions } \\
\text { were fulfilled }\end{array}$ & References \\
\hline CR lumbar spine & $\begin{array}{l}\text { Any of the following abnormalities on CR of the lumbar spine: } \\
\text {-Any fracture of the vertebral body or spinous or transverse processes } \\
\text {-Spinal malalignment }\end{array}$ & {$[20,47]$} \\
\hline $\begin{array}{l}\text { CR pelvis and abdominal } \\
\text { ultrasonography }\end{array}$ & $\begin{array}{l}\text { Any of the following pelvic fractures on CR: } \\
\text {-Pubic bone fracture } \\
\text {-Fracture acetabulum } \\
\text {-Fracture illiac wing } \\
\text {-Luxation sacro-iliac joint } \\
\text {-Fracture sacrum } \\
\text {-Femoral head fracture } \\
\text {-Symphysiolysis } \\
\text {-Luxation hip } \\
\text { Abnormal abdominal ultrasound: } \\
\text {-Presence of free fluid }\end{array}$ & {$[20,21,37]$} \\
\hline $\mathrm{BE}<-3$ & -Arterial blood gas base excess less than $-3 \mathrm{mmol} / 1$ in initial blood gas samples & \\
\hline $\mathrm{Hb}<6$ & -Blood plasma haemoglobin concentration less than $6 \mathrm{mmol} / \mathrm{l}$ & \\
\hline
\end{tabular}

Note: ${ }^{a}$ ED, emergency department; ${ }^{b} \mathrm{PE}$, physical examination; ${ }^{\mathrm{c}} \mathrm{CR}$, conventional radiography

spine was not performed or was only obtained in an anteroposterior direction.

Table 2 shows the definitions of positive composite predictors. Table 3 shows the univariate relationships between these predictors and presence of any chest injuries on CT. This table indicates that only "dangerous mechanism of injury" and "PE abdomen" failed to demonstrate a statistically significant crude odds ratio. After multivariate logistic regression analysis on the remaining 13 composite predictors, 9 independent predictors significantly con-
Fig. 1 Diagram illustrating patient flow for study selection and the number of patients with chest injuries on $\mathrm{CT}$, occult chest injuries on $\mathrm{CT}$ and occult injuries on CT with an impact on patient management. CR, conventional radiography of the chest and thoracic spine; CT, computed tomography; occult injuries, injuries that were only detected on $\mathrm{CT}$, but not on $\mathrm{CR}$

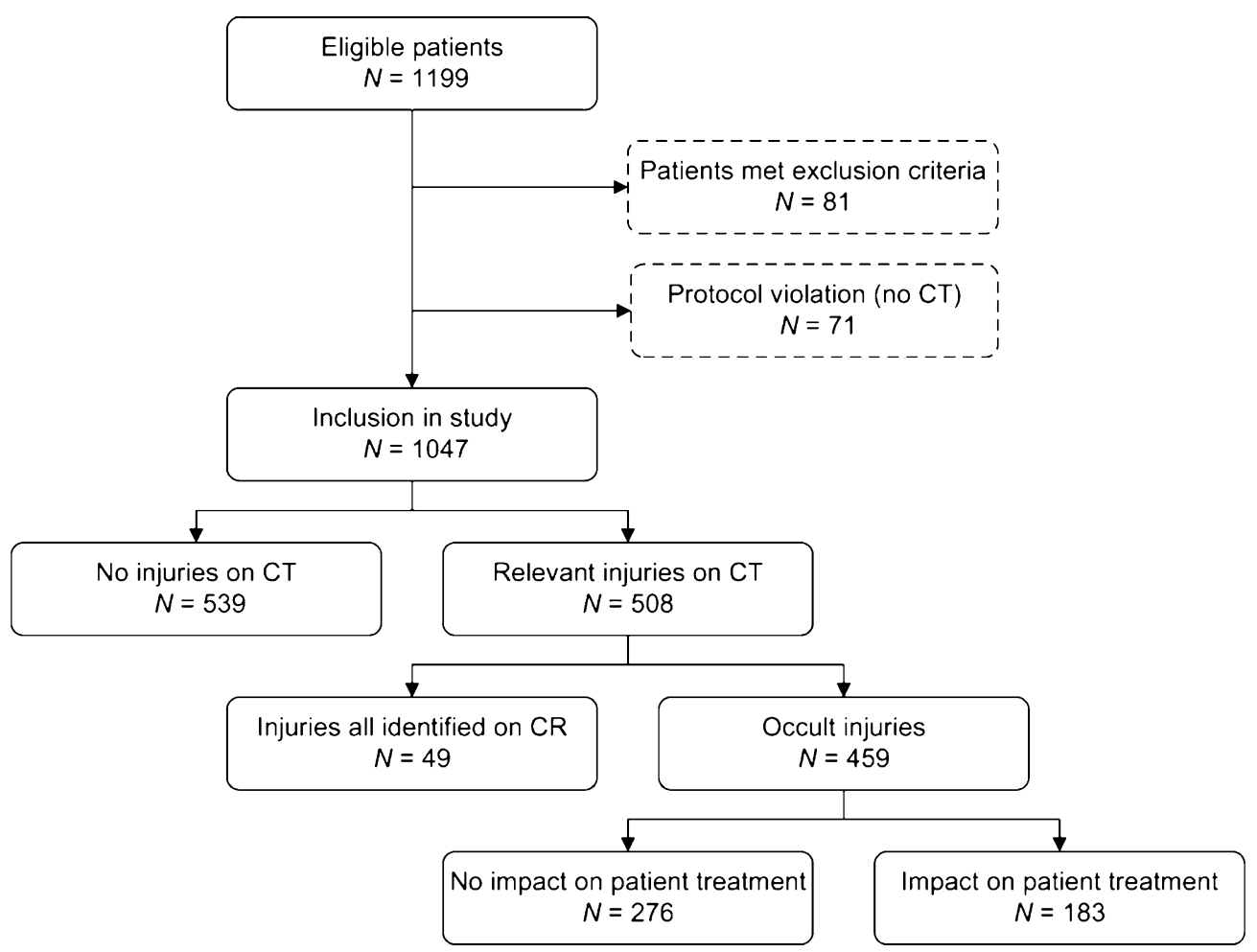


Table 3 Univariate relationships between positive predictors and the presence of any chest injuries on CT

\begin{tabular}{lll}
\hline Positive composite predictors & OR $(95 \%$ CI) & $p$ value \\
\hline$\geq 55$ years $(n=208)$ & $2.37(1.73-3.25)$ & $<0.001$ \\
Dangerous mechanism of injury $(\mathrm{n}=235)$ & $1.22(0.91-1.63)$ & 0.209 \\
PE chest $(\mathrm{n}=361)$ & $4.64(3.5-6.3)$ & $<0.001$ \\
PE circulatory problems $(\mathrm{n}=184)$ & $2.58(1.84-3.61)$ & $<0.001$ \\
PE altered sensorium $(\mathrm{n}=395)$ & $2.54(1.97-3.29)$ & $<0.001$ \\
PE supraclavicular injury $(\mathrm{n}=615)$ & $1.79(1.40-2.30)$ & $<0.001$ \\
PE thoracic spine $(\mathrm{n}=134)$ & $1.51(1.05-2.18)$ & 0.027 \\
PE abdomen (n=175) & $1.18(0.85-1.64)$ & 0.313 \\
PE extremity fracture $(\mathrm{n}=514)$ & $1.40(1.09-1.78)$ & 0.008 \\
CR chest $(\mathrm{n}=366)$ & $15.6(11.12-21.93)$ & $<0.001$ \\
CR thoracic spine $(\mathrm{n}=129)$ & $2.55(1.72-3.77)$ & $<0.001$ \\
CR lumbar spine $(\mathrm{n}=86)$ & $2.64(1.64-4.26)$ & $<0.001$ \\
CR pelvis and abdominal ultrasonography $(\mathrm{n}=209)$ & $<0.001$ \\
BE $<-3$ positive $(\mathrm{n}=351)$ & $2.89(2.09-3.99)$ & $<0.001$ \\
Hb $<6$ (n=51) & $3.81(2.89-5.01)$ & $<0.001$
\end{tabular}

Notes: OR, crude odds ratio; $95 \%$ CI, 95\% confidence interval

Definitions of positive composite predictors are displayed in Table 2

tributed to the prediction of presence of chest injuries on CT (Table 4): Abnormal CR of the chest, abnormal chest $\mathrm{PE}, \mathrm{BE}<-3 \mathrm{mmol} / \mathrm{l}$, abnormal abdominal ultrasonography or pelvic $\mathrm{CR}$, abnormal thoracic spine $\mathrm{PE}$, age $\geq 55$ years, $\mathrm{Hb}<6$, abnormal $\mathrm{CR}$ of the thoracic spine and altered sensorium.

Figure 2 shows the ROC curve of the predictive model containing these nine predictors with an R-square of 0.478 and an AUC of 0.85 (95\% CI: 0.83-0.87). After bootstrap analysis, the corrected R-square was 0.455 and the corrected AUC was 0.71. The ROC curve in Fig. 2 includes the cutoff point at which patients were stratified

Table 4 Independent predictors of the presence of any chest injuries on $\mathrm{CT}$

\begin{tabular}{ll}
\hline Positive composite predictors & Adjusted OR $(95 \% \mathrm{CI})$ \\
\hline$\geq 55$ years & $1.6(1.1-2.4)$ \\
PE chest & $3.0(2.2-4.2)$ \\
PE of the thoracic spine & $1.8(1.1-2.8)$ \\
PE altered sensorium & $1.5(1.0-2.1)$ \\
CR chest & $9.4(6.5-14)$ \\
CR thoracic spine & $1.7(1.1-2.9)$ \\
CR pelvis and abdominal ultrasonography & $2.3(1.5-3.4)$ \\
$\mathrm{BE}<-3$ & $2.0(1.4-2.9)$ \\
$\mathrm{Hb}<6$ & $2.9(1.1-7.6)$ \\
\hline
\end{tabular}

Note: OR, odds ratio adjusted to all other predictors in the model; CI, confidence interval. Definitions of positive composite predictors are displayed in Table 2 into low-risk and high-risk patients. Of all included patients, $855(82 \%)$ patients had one or more positive predictors and were classified as high-risk patients. One hundred ninety-two patients $(18 \%)$ had no positive predictor and were classified as low-risk patients.

Of all 508 patients with injuries on CT, our model correctly classified 484 patients as high-risk patients (sensitivity: 0.95 ; 95\% CI, 0.93-0.97) and the remaining 24 patients with injuries on CT as low-risk patients. This

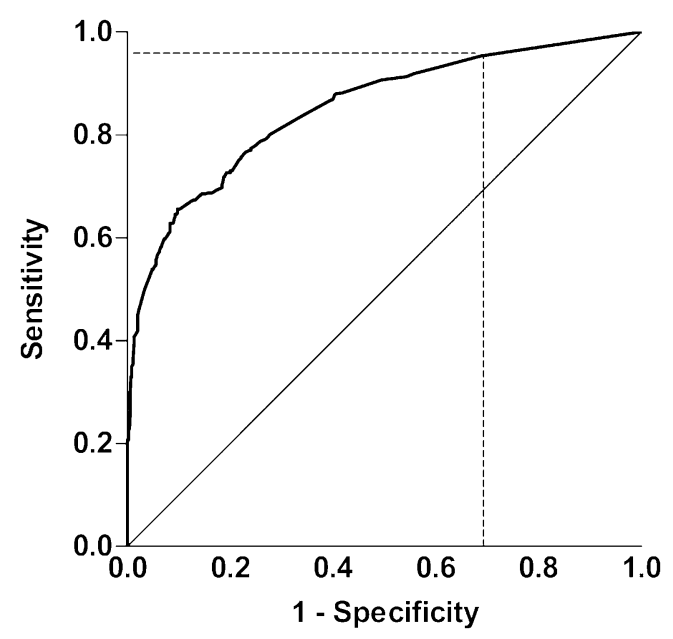

Fig. 2 Receiver-operating characteristic (ROC) curve of the predictive model containing nine predictors of injuries on chest CT. The cutoff point (dashed lines) is located at a sensitivity of 0.95 and at a specificity of 0.31 . (Area under the curve $=0.85 ; 95 \%$ confidence interval, $0.83-0.87$ ) 
means that the probability of having CT injuries in the lowrisk patient group was $24 / 192=13 \%(95 \%$ CI: 9-18\%). These patients mainly had minimal pulmonary contusion, minimal pneumothoraces, one or two rib fractures and scapular fractures (Table 5). The model correctly classified patients without injuries on CT $(\mathrm{n}=539)$ as low-risk in 168 patients (specificity: $0.31 ; 95 \%$ CI, 0.27-0.35).

Of all 183 patients with clinically relevant occult injuries on CT, 179 were correctly classified as high risk (sensitivity; $0.98,95 \%$ CI $0.96-1$ ). Four out of 192 low-risk patients $(2 \%$; $95 \%$ CI $1-5 \%)$ had clinically relevant occult injuries: one patient had a stable fracture of the XII vertebral body that was only visualised on $\mathrm{CR}$ of the lumbar spine, but not on $\mathrm{CR}$ of the thoracic spine. This patient was treated conservatively with a brace. A second patient had pulmonary contusion, one rib fracture and a pneumothorax of moderate size that were not visualised on chest CR. These injuries were treated conservatively with close observation. Although in this patient none of the nine predictors was positive, cervical spine CT demonstrated subcutaneous emphysema. A third patient who was classified as belonging to the low-risk patient group had three rib fractures. A fourth low-risk patient had a small pneumothorax with pulmonary contusion. These latter patients were closely observed without further surgical interventions. None of the low-risk-group patients suffered from aortic injury, diaphragmatic injury, haemothoraces or large pneumothoraces (Table 5).

\section{Discussion}

In this prospective study, we derived a set of variables that predict whether $\mathrm{CT}$ of the chest including the thoracic spine is likely to reveal relevant injuries in high-energy blunt trauma patients. These clinically intuitive predictors were derived from data that are available at initial presentation in the emergency department, including age, physical examination, laboratory analyses, CR and abdominal ultrasonography. If CTs were obtained in patients with one or more positive predictors (high-risk patients) only, CT investigations would have been avoided in $18 \%$ of patients in this study's population, thereby decreasing ionising radiation exposure and health-care expenditure.

However, our study data also suggested that if our positive predictors were implemented as scanning indications, 5\% (24/508) of all patients with chest injuries on CT would not be identified. This implies that the chance of missing injuries of

Table 5 Prevalence of distinct chest injuries on CT in all patients, in patients who had $\geq 1$ positive predictor in the predictive model (highrisk patients) and in patients who had no positive predictor of chest injury (low-risk patients)

\begin{tabular}{|c|c|c|c|c|c|c|}
\hline \multirow{2}{*}{$\begin{array}{l}\text { Injuries on } \mathrm{CT} \\
\text { Pneumothorax }\end{array}$} & \multicolumn{2}{|c|}{$\begin{array}{l}\text { No. }(\%) \text { of patients } \\
(n=1047)\end{array}$} & \multicolumn{2}{|c|}{$\begin{array}{l}\text { No. }(\%) \text { of high-risk patients } \\
(n=855)\end{array}$} & \multicolumn{2}{|c|}{$\begin{array}{l}\text { No. }(\%) \text { of low-risk patients } \\
(n=192)\end{array}$} \\
\hline & 234 & $(22.3)$ & 228 & $(26.6)$ & 6 & $(3.1)$ \\
\hline -Moderate pneumothorax & 90 & $(8.6)$ & 89 & $(10.4)$ & 1 & $(0.5)$ \\
\hline -Severe pneumothorax & 35 & $(3.3)$ & 35 & $(4.1)$ & 0 & $(0.0)$ \\
\hline Haemothorax & 58 & $(5.5)$ & 58 & $(6.8)$ & 0 & $(0.0)$ \\
\hline Pulmonary contusion & 288 & $(28)$ & 173 & $(20.2)$ & 15 & $(7.8)$ \\
\hline -Moderate or severe contusion & 71 & $(6.8)$ & 71 & $(8.3)$ & 0 & $(0.0)$ \\
\hline Oesophageal injury & 1 & $(0.1)$ & 1 & $(0.1)$ & 0 & $(0.0)$ \\
\hline Tracheobronchial injury & 2 & $(0.2)$ & 2 & $(0.2)$ & 0 & $(0.0)$ \\
\hline Aortic injury & 9 & $(0.8)$ & 9 & $(1.1)$ & 0 & $(0.0)$ \\
\hline Injury to the subclavian vein & 1 & $(0.1)$ & 1 & $(0.1)$ & 0 & $(0.0)$ \\
\hline Rib fracture & 317 & $(30.3)$ & 311 & $(36.4)$ & 6 & $(3.1)$ \\
\hline$->2$ Rib fractures & 233 & $(22.2)$ & 232 & $(27.1)$ & 1 & $(0.5)$ \\
\hline Scapular fracture & 76 & $(7.3)$ & 73 & $(8.5)$ & 3 & $(1.5)$ \\
\hline Sternal fracture & 51 & $(4.9)$ & 51 & $(6.0)$ & 0 & $(0.0)$ \\
\hline Diaphragmatic injury & 5 & $(0.5)$ & 5 & $(0.6)$ & 0 & $(0.0)$ \\
\hline Any thoracic spinal fracture & 123 & $(12)$ & 122 & $(14.2)$ & 1 & $(0.5)$ \\
\hline -Vertebral body fracture & 81 & $(7.7)$ & 80 & $(9.4)$ & 1 & $(0.5)$ \\
\hline -Transverse process fracture & 59 & $(5.6)$ & 59 & $(6.9)$ & 0 & $(0.0)$ \\
\hline -Spinous process fracture & 20 & $(1.9)$ & 20 & $(2.3)$ & 0 & $(0.0)$ \\
\hline Total (any chest injury) & 508 & $(48.5)$ & 484 & (56.6) & 24 & (12.5) \\
\hline
\end{tabular}

Notes: Numbers in parentheses are percentages of patient groups. A patient could have multiple chest injuries 
the chest remains $13 \%(24 / 192)$ in the low-risk patient group if these patients do not undergo chest CT. This risk is substantially lower compared with chest injury risk in the entire blunt trauma population, which was $49 \%$ in our study; it is even relatively low compared with previously described "low-risk" populations. Omert et al. reported a prevalence of $39 \%(95 \%$ CI, $27-51 \%)$ of chest injuries in patients with normal CR and normal physical examination [17], and Salim et al. reported a prevalence of $20 \%(95 \%$ CI, $16-23 \%)$ of pulmonary, mediastinal and rib injuries in patients who were clinically evaluable and had both a normal physical examination and CR [2].

One may pose the question of whether an injury probability of $13 \%$ is acceptable for a low-risk patient group. We argue that this risk can be considered acceptable, mainly because these chest injuries had no clinically relevance in most cases. The small pulmonary contusions, pneumothoraces and rib fractures rarely had an impact on patient management (in only $2 \%$ of all low-risk patients) and were, perhaps with the exception of the missed thoracic spine fracture, unlikely to affect patient morbidity if left unmanaged. Although costeffectiveness studies have established acceptable risks for cost-effective injury detection by using CT $[18,19]$, these, unfortunately, do not pertain to injuries of the entire chest including the thoracic spine.

Predicting variables that were evaluated in this study were, in part, based on previous studies on appropriate patient selection for chest CT. However, these studies only investigated distinct chest or thoracic injuries and used a case-control design [20,21], or did not use CT as a standard of reference [14, 22-24].

To our knowledge, this was one of the first prospective studies to identify selection criteria to facilitate a more appropriate use of CT of the entire chest in adult blunt trauma patients. We used CT as the standard of reference in all included patients. We investigated and described strong criteria that predict presence of any type of relevant chest injuries on CT. Our results might not be surprising as they indicate that chest $\mathrm{CT}$ is warranted with abnormal PE or CR. However, this study adds to previous knowledge by defining not only in which patients chest CT is warranted, but also by defining in which patients chest CT could be safely omitted. With further validation, incorporation of these criteria into a diagnostic algorithm for patient selection for chest CT could be an important step towards optimising resource use in trauma imaging.

We are aware that several centres do not use CR of the spine because it is not as sensitive as CT in injury detection [25] or do not have laboratory analyses available in the emergency department. However, omission of these tests from our prediction model substantially decreased predictive capacity. As long as no techniques other than CR of the spine and laboratory analyses are used to provide indications for CT imaging, these investigations seem indispensible for selective chest $\mathrm{CT}$ algorithms in patients who do not have other positive predictors.
Our study has a number of limitations. According to the Oxford levels of evidence grading [26], good diagnostic research incorporates index tests and reference tests that are applied blindly and objectively. However, the standard of reference (CT) was not interpreted independently from other clinical information because in our practice, radiologists and surgeons work closely together in trauma patient care. However, we do not consider this a major source of incorporation bias because chest $\mathrm{CT}$ rarely misses injuries that are visualised on CR [27].

A second limitation is that we abstracted information on potential predictors or index tests from medical records. Although we used objective predictor definitions and instructed trauma team members to record all data on potential predictors prospectively (blinded to CT outcomes), this introduced a retrospective component in this study. However, we minimised hindsight bias on presence of predictors by dictating which data on potential predictors had to be present in all medical records and by personally monitoring patient evaluations. Researchers, therefore, only rarely had to ask trauma team members for additional information on potential predictors in retrospect.

Third, the findings of our study have not been externally validated in different trauma populations and settings. External validation is needed for three reasons:

1. The performance of our model is likely to be overoptimistic: The model was created in the same sample of patients in which the performance was determined and should therefore be evaluated in a new sample of trauma patients in our centre.

2. External validation should furthermore be carried out to evaluate our model in other centres: The definition of some clinically relevant injuries (such as occult injuries for which the care level was upgraded or additional diagnostic work-up was needed) is institution- and clinician-dependent.

3. The costs, effectiveness and impact on patient quality of life of CT in chest injury diagnosis should be further investigated.

\section{Conclusions}

In conclusion, significant independent predictors of injuries on chest CT in high-energy blunt trauma patients are age $\geq 55$ years, abnormal chest PE, altered sensorium, abnormal thoracic spine PE, abnormal chest and thoracic spine CR, abnormal abdominal US or pelvic $\mathrm{CR}, \mathrm{Hb}<6$ and $\mathrm{BE}<$ $-3 \mathrm{mmol} / \mathrm{l}$. Presence of any of these criteria can predict presence of chest injuries on CT with a sensitivity of $95 \%$. If CT is omitted in patients without any of these criteria, the number of CT investigations can be substantially reduced, while the risk of missing relevant injuries with clinical importance seems to remain low. Although our findings need external validation, a diagnostic algorithm employing these 
criteria has the potential to reduce unnecessary CT examinations of the chest in blunt trauma patients in the future.

Acknowledgements We thank Yvonne L. Hoogeveen, $\mathrm{PhD}$, for prereading the manuscript.
Open Access This article is distributed under the terms of the Creative Commons Attribution Noncommercial License which permits any noncommercial use, distribution, and reproduction in any medium, provided the original author(s) and source are credited.

\section{References}

1. Aucar JA, Fernandez L, Wagner-Mann $\mathrm{C}$ (2007) If a picture is worth a thousand words, what is a trauma computerized tomography panel worth? Am J Surg 194:734-739

2. Salim A, Sangthong B, Martin M, Brown C et al (2006) Whole body imaging in blunt multisystem trauma patients without obvious signs of injury: results of a prospective study. Arch Surg 141:468-473

3. Broder J, Warshauer DM (2006) Increasing utilization of computed tomography in the adult emergency department, 2000-2005. Emerg Radiol 13:25-30

4. Brenner DJ, Hall DP (2007) Computed tomography-an increasing source of radiation exposure. N Engl J Med 357:2277-2284

5. Amis ES Jr, Butler PF, Applegate KE et al (2007) American College of Radiology white paper on radiation dose in medicine. J Am Coll Radiol 4:272-284

6. Heyer CM, Rduch GJ, Wick M et al (2005) [Evaluation of multiple trauma victims with 16-row multidetector $\mathrm{CT}$ (MDCT): a time analysis]. Röfo 177:1677-1682

7. Brink M, Kool DR, Dekker HM et al (2009) Predictors of abnormal chest CT after blunt trauma: a critical appraisal of the literature. Clin Radiol. 64(3):272283

8. American College of Radiology (2005) ACR Appropriateness Criteria Suspected aortic injury. Available at: http://acsearch.acr.org/default.aspx. Accessed 19 Jan 2009

9. American College of Radiology (2007) ACR Appropriateness Criteria suspected spine trauma. Available at: http://acsearch.acr.org/default.aspx. Accessed 19 Jan 2009

10. Eastern Association for the Surgery of Trauma (2006) Practice Management Guidelines for the Screening of Thoracolumbar Spine Fracture. Available at: http://www.east.org/tpg/ tlspine.pdf. Accessed 19 Jan 2009
11. Nagy K, Fabian T, Rodman G et al (2000) Guidelines for the diagnosis and management of blunt aortic injury: an EAST Practice Management Guidelines Work Group. J Trauma 48:11281143

12. Brink M, Deunk J, Dekker HM et al (2008) Added value of routine chest MDCT after blunt trauma: evaluation of additional findings and impact on patient management. AJR Am J Roentgenol 190:1591-1598

13. American College of Surgeons Committee on Trauma (2004) Advanced trauma life support program for doctors, 7th edn. American College of Surgeons, Chicago

14. Hsu JM, Joseph T, Ellis AM (2003) Thoracolumbar fracture in blunt trauma patients: guidelines for diagnosis and imaging. Injury 34:426-433

15. Hosmer D, Lemeshow S (2000) Modelbuilding strategies and methods for logistic regression. In: Cressie N, Fisher N, Johnstone I et al (eds) Applied logistic regression. 2nd edn. Wiley-Interscience, New York, pp 91-142

16. Harrel FE (2001) Regression modeling strategies: with applications to linear models, logistic regression and survival analysis, 1st edn. Springer, New York

17. Omert L, Yeaney WW, Protetch J (2001) Efficacy of thoracic computerized tomography in blunt chest trauma. Am Surg 67:660-664

18. Blackmore CC, Ramsey SD, Mann FA et al (1999) Cervical spine screening with CT in trauma patients: a costeffectiveness analysis. Radiology 212:117-125

19. Hunink MG, Bos JJ (1995) Triage of patients to angiography for detection of aortic rupture after blunt chest trauma: cost-effectiveness analysis of using CT. AJR Am J Roentgenol 165:27-36

20. Blackmore CC, Zweibel A, Mann FA (2000) Determining risk of traumatic aortic injury: how to optimize imaging strategy. AJR Am J Roentgenol 174:343-347

21. Kirkham JR, Blackmore CC (2007) Screening for aortic injury with chest radiography and clinical factors. Emerg Radiol 14:211-217
22. Holmes JF, Sokolove PE, Brant WE et al (2002) A clinical decision rule for identifying children with thoracic injuries after blunt torso trauma. Ann Emerg Med 39:492-499

23. Gittelman MA, Gonzalez-del-Rey J, Brody AS et al (2003) Clinical predictors for the selective use of chest radiographs in pediatric blunt trauma evaluations. J Trauma 55:670-676

24. Holmes JF, Panacek EA, Miller PQ et al (2003) Prospective evaluation of criteria for obtaining thoracolumbar radiographs in trauma patients. J Emerg Med 24:1-7

25. Inaba $\mathrm{K}$, Munera $\mathrm{F}, \mathrm{McKenney} \mathrm{M}$ et al (2006) Visceral torso computed tomography for clearance of the thoracolumbar spine in trauma: a review of the literature. J Trauma 60:915-920

26. Wright JG (2007) A practical guide to assigning levels of evidence. J Bone Joint Surg Am 89:1128-1130

27. Moons KG, Grobbee DE (2002) When should we remain blind and when should our eyes remain open in diagnostic studies? J Clin Epidemiol 55:633-636

28. Plurad D, Green D, Demetriades D et al (2007) The Increasing use of chest computed tomography for trauma: is it being overutilized? J Trauma 62:631635

29. Ungar TC, Wolf SJ, Haukoos JS et al (2006) Derivation of a clinical decision rule to exclude thoracic aortic imaging in patients with blunt chest trauma after motor vehicle collisions. J Trauma 61:1150-1155

30. Dyer DS, Moore EE, Ilke DN et al (2000) Thoracic aortic injury: how predictive is mechanism and is chest computed tomography a reliable screening tool? A prospective study of 1561 patients. J Trauma 48:673-682

31. Nirula R, Talmor D, Brasel K (2005) Predicting significant torso trauma. J Trauma 59:132-135

32. Frankel HL, Rozycki GS, Ochsner MG et al (1994) Indications for obtaining surveillance thoracic and lumbar spine radiographs. J Trauma 37:673-676 
33. Rodriguez RM, Hendey GW, Marek G et al (2006) A pilot study to derive clinical variables for selective chest radiography in blunt trauma patients. Ann Emerg Med 47:415-418

34. Chen SC, Chang KJ, Hsu CY (1998) Accuracy of auscultation in the detection of haemopneumothorax. Eur J Surg 164:643-645

35. Ayed AK, Al-Shawaf E (2004) Diagnosis and treatment of traumatic intrathoracic major bronchial disruption. Injury 35:494-499

36. Barton ED, Epperson M, Hoyt DB et al (1995) Prehospital needle aspiration and tube thoracostomy in trauma victims: a six-year experience with aeromedical crews. J Emerg Med 13:155-163

37. Traub M, Stevenson M, McEvoy S et al (2007) The use of chest computed tomography versus chest X-ray in patients with major blunt trauma. Injury 38:43-47
38. Tam MM (2005) Occult pneumothorax in trauma patients: should this be sought in the focused assessment with sonography for trauma examination? Emerg Med Australas 17:488-493

39. Bokhari F, Brakenridge S, Nagy K et al (2002) Prospective evaluation of the sensitivity of physical examination in chest trauma. J Trauma 53:1135-1138

40. Wisbach GG, Sise MJ, Sack DI et al (2007) What is the role of chest X-ray in the initial assessment of stable trauma patients? J Trauma 62:74-78

41. Demetriades D, Gomez H, Velmahos GC et al (1998) Routine helical computed tomographic evaluation of the mediastinum in high-risk blunt trauma patients. Arch Surg 133:1084-1088

42. Meldon SW, Moettus LN (1995) Thoracolumbar spine fractures: clinical presentation and the effect of altered sensorium and major injury. J Trauma 39:1110-1114

43. Chang CH, Holmes JF, Mower WR et al (2005) Distracting injuries in patients with vertebral injuries. J Emerg Med $28: 147-152$
44. Stanislas MJ, Latham JM, Porter KM et al (1998) A high risk group for thoracolumbar fractures. Injury 29:15-18

45. Exadaktylos AK, Sclabas G, Schmid SW et al (2001) Do we really need routine computed tomographic scanning in the primary evaluation of blunt chest trauma in patients with "normal" chest radiograph? J Trauma 51:11731176

46. Marts B, Durham R, Shapiro M et al (1994) Computed tomography in the diagnosis of blunt thoracic injury. Am J Surg 168:688-692

47. Berry GE, Adams S, Harris MB et al (2005) Are plain radiographs of the spine necessary during evaluation after blunt trauma? Accuracy of screening torso computed tomography in thoracic/ lumbar spine fracture diagnosis. J Trauma 59:1410-1413 